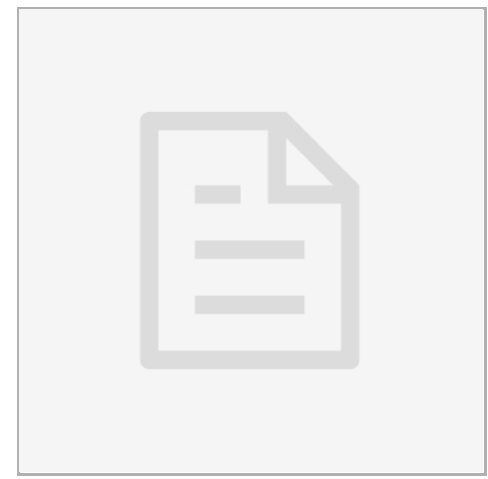

VERSION 2

open Access

DOI:

dx.doi.org/10.17504/protocol s.io. 2 dzga76

Protocol Citation: Matema LE Imakumbili 2019.

Determination of total hydrogen cyanide levels in fresh cassava roots using the picrate paper method. protocols.io https://dx.doi.org/10.17504/p rotocols.io. $2 \mathrm{dzga} 76$

License: This is an open access protocol distributed under the terms of the Creative Commons Attribution License, which permits unrestricted use, distribution, and reproduction in any medium, provided the original author and source are credited

Protocol status: Working We use this protocol and it's working

Created: Apr 27, 2019

Last Modified: Apr 28, 2019

PROTOCOL integer ID:

22681

\section{(3) Determination of total hydrogen cyanide levels in fresh cassava roots using the picrate paper method V.2}

Matema LE Imakumbili ${ }^{1}$

${ }^{1}$ Sokoine University of Agriculture

Matema LE Imakumbili

Sokoine University of Agriculture 


\section{ABSTRACT}

This protocol describes how to analyse total cyanide $(\mathrm{HCN})$ levels in fresh cassava roots using the picrate paper method. The method gives the potential cyanide content of cassava roots on a fresh weight basis. The picrate paper method for root cyanide determination relies on endogenous enzymes to breakdown the cyanogenic glucosides (linamarine) in fresh cassava root samples. The hydrogen cyanide liberated in the process reacts with picrate papers that have a colour pigment which darkens with released cyanide. The variations in the darkening of picrate papers is then used to measure the amount of hydrogen cyanide released from fresh cassava root samples. The picrate paper method is argued to not be a very reliable method for cassava cyanide determination. However, if you are going to have to work far from a laboratory where you cannot perform another preferred method, then the picrate paper method is your best option as you may not be able to avoid root deterioration and cyanide loss during transportation of cassava root samples. The key to using this method is focus, precision and timeliness. If these three are maintained throughout the analysis then chances of introducing errors are reduced making the obtained results more comparable with other cyanide determination methods percieved as more accurate.

This protocol has been already well outlined by $\mathrm{Dr}$ J.H. Bradbury and his team, so please refer to their document (see the attached document below). A kit for cyanide analysis in cassava roots called PROTOCOL A: DETERMINATION OF TOTAL CYANIDE IN CASSAVA ROOTS, has also been designed by them. This protocol describes how the Protocol A kit can be used to determine cyanide in cassava roots. This protocol is not a replacement of the original protocol. Some of the contents of this protocol have however been directly copied from the original protocol; they are indicated in bold text. This protocol however contains additional insights from things encountered during the use of the kit; this information is in normal text. In addition to this, this protocol includes insights that Dr Bradbury shared to help troubleshoot a few unforeseen issues; these insights are in italics.

\section{Protocol A.pdf}

\section{GUIDELINES}

When not in use, picrate papers need to be kept in the deep freezer where they remain stable indefinitely. Place the picrate papers (still in their original packaging) into a Ziploc plastic bag and then place the Ziploc plastic bag with its contents into a well-sealed firm opaque plastic storage container (a lunch box would be fine). This protects the picrate papers from being crushed and also keeps them away from light.

Picrate papers must be kept dry! Make sure that the deep freezer works very well 
and not as a freezer. Freezers have a tendency of wetting picrate papers, making them unusable. If the refrigerator you are using has a freezing compartment and not a deep freezing compartment, then do not place the picrate papers in the freezing compartment. Instead, place the picrate papers in the non-freezing compartment (refrigerator). Also use the non-freezing compartment when you are not sure if the refrigerator has a deep freezing function. The non-freezing compartment will not freeze the picrate papers but will at least keep them dry. It will also keep them cool thus delaying darkening.

Picrate papers become dark when stored at room temperatures or when exposed to sunlight. It takes about 1 month for picrate papers to darken when they are stored at room temperature. After this they cannot be used with the colour chart but are ok with the spectrometer because the colour cancels out. When they have not been used yet, picrate papers must always retain their original light yellow colour. The standard 50 ppm papers, which are pink in colour, must be stored in the refrigerator as the level decreases gradually over time.

You will sometimes have to use the picrate papers away from a lab. This could happen if the field from which the cassava roots will be sampled is far away. In such a situation the timely transportation of root samples back to the lab would be impossible. Hence a number of things must be done if you will be working away from the lab as follows:

1. While travelling always place the picrate papers and standard pink linamarin papers in a cool box with ice packs. The picrate papers should still be in the firm opaque storage container they had been placed in, it is convenient to carry them in this way. Also place the small bottle containing the standard pink linamarin in the same storage box as the picrate papers.

2. Try to arrange for a deep freezer and a refrigerator at your destination. If this is not possible then you will have to continue using the cool box. You could use special ice packs to help keep the cooler cool, some can remain frozen for days.

3. Try to also arrange for a room where you can conduct your analyses. If it is all office space bear in mind that people may not welcome your root samples, so please be understanding.

4. Make sure you have a hard surface to work on, for cutting.

5. If you can arrange that the room where you shall place the fresh root samples before analysing them has air-conditioning this would be good in helping keep the roots fresh. The place where you shall incubate the samples should however not have air-conditioning.

6. Arrange for a dark cupboard or place where you can incubate your samples in the dark, without interference.

7. Don't forget to carry one large knife and a small knife for root cutting. Also carry a large basin as you may have to wash the plastic bottles in order to re-use them. 
Picrate papers need to be all treated the same way. The time they spend inside and outside the deep freezer or in the cool box must be all the same. In this way the picrate papers will lose colour in a similar manner (not that you want them to lose colour). Thus, if a batch of picrate papers has been differently treated, then it should be treated as one. This means that each batch of picrate papers that has been treated a certain way must have its own blank. Since spectrophotometer readings are only done later on, then a few unused picrate papers must be kept from each batch so as to serve as blanks for the samples that used picrate papers from that particular batch. At least two of these should be kept in addition to the picrate paper used to test the blank during analyses.

\section{MATERIALS}

- 1 large knife

- 1 small knife

- A small disposable plastic knife

- Marker pens (both fine and thick tip)

- 1 or 2 basins (for washing and drying plastic bottles for their reuse)

- $1 \mathrm{~L}$ bottled water

- Distilled water

- Thermometer

- Gladwrap or tiny sized plastic bags

- Test tubes or 25 - $50 \mathrm{ml}$ beakers

Protocol A, which gives full instructions for total cyanide analysis of cassava roots.

1. A plastic balance with a $\mathbf{1 0 0} \mathbf{~ m g}$ weight glued into one spoon, for weighing $100 \mathrm{mg}$ of cassava root.

2. $\mathbf{3 0}$ flat-bottomed plastic bottles with screw lids.

3. Two graduated $1 \mathrm{ml}$, plastic pipettes.

4. Bottle containing $\mathbf{1 0 0}$ buffer papers at pH 6 .

5. $\mathbf{1 0 0}$ yellow picrate papers glued to strips of clear plastic with hobby glue.

6. Colour chart with $\mathbf{1 0}$ shades of colour which correspond to $0-800 \mathrm{ppm}$ total cyanide.

7. Ten pink standard papers containing linamarin equal to $50 \mathrm{ppm}$ cyanide.

8. Ten paper discs which contain buffer at $\mathrm{pH} 6$ and the enzyme linamarase. These papers have a small black spot, so that they will not be confused with buffer papers.

9. Filter paper and plastic overhead transparency sheets for making more picrate papers. 


\section{BEFORE START INSTRUCTIONS}

The protocol has been designed to be used on cassava roots that are still in their fresh state. It can also be used on frozen cassava root samples that are still in their fresh state. This protocol should not be used to analyse the cyanide content of cassava flour, cassava leaves or of any other processed cassava product. These have their own specially designed test kits.

For the results to be accurate ensure that the quality of roots are as close as possible to the state they were in right after harvest.

Place the small plastic balance on its $U$-shaped mount so that it swings freely. It has a $100 \mathrm{mg}$ weight glued inside one spoon.

Notes:

- The small plastic balance is a useful tool for weighing the needed amount of cassava root sample. If used correctly it is an accurate tool. Exercise patience when using it to weigh your samples and learn to use it quickly and with precision. Carrying out this step quickly will lessen the time that roots are left exposed to air, which will reduce cyanide losses.

- If you are working from a lab you can use a $0.0001 \mathrm{~g}(0.1 \mathrm{mg})$ digital analytical balance to weigh your sample. Some labs will however not have balances that can accurately weigh weights as small as $100 \mathrm{mg}$. In this case the small balance should still be used.

2 A 1-2 $\mathrm{mm}$ thick cross section of the clean cassava root is cut across the middle of the root. The peel is removed and a sector is cut and its weight adjusted to $100 \mathrm{mg}$ by cutting off small pieces along the straight edge of the sector and weighing on the small balance.

\section{Notes:}

- See diagram from original document for demonstration.

- This is the step where you will have to use the knives for cutting. The large knife is best for cutting roots in half and for taking a slice of its cross section. The small knife is best for cutting out the root sector.

- Sometimes roots are very large and a sector cut in this manner will weigh more than $100 \mathrm{mg}$ no matter its size. When this is the case cut out small sections along the sector to get an average representation of $\mathrm{HCN}$ levels across the sector. See Fig 1. 


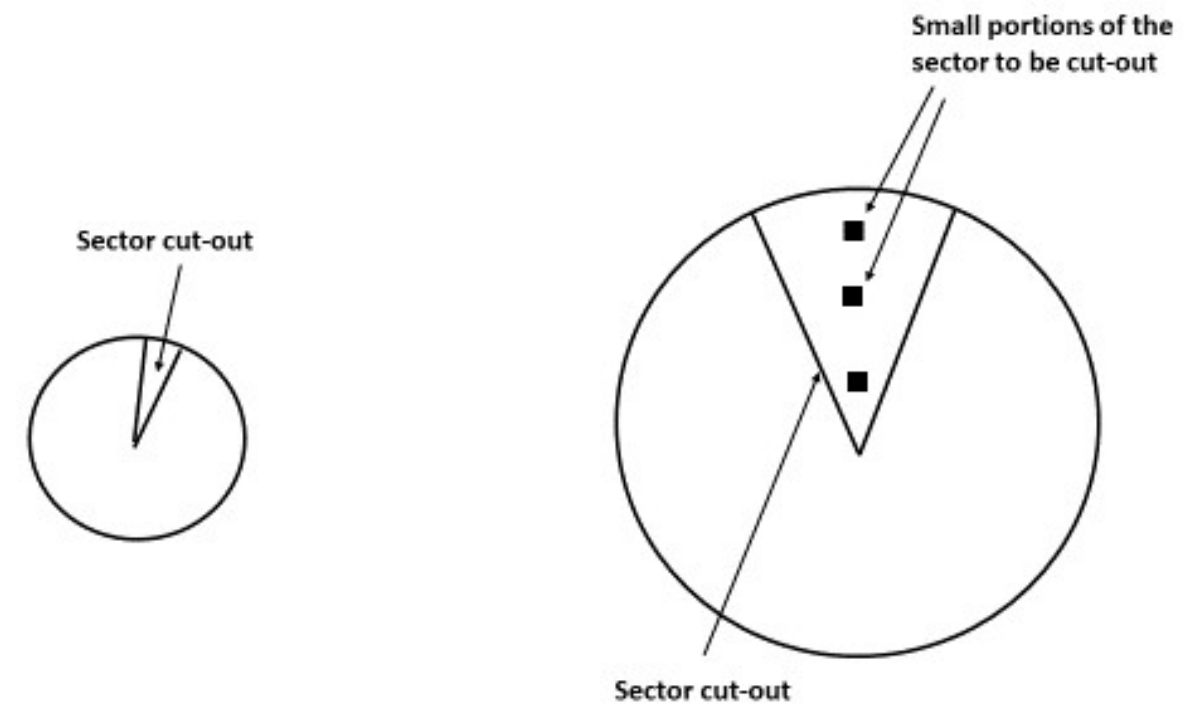

Figure 1. Sector cut from small root sample (left); Portions of sector to be cut from large root sample (right)

3 Place a round paper disc containing buffer at pH 6 in a flat-bottomed plastic bottle and place a $100 \mathrm{mg}$ sector of cassava root on top of it. Note. Do not leave these sectors standing in the air for more than one hour before setting up the analysis.

\section{Notes:}

- Once a sector of one root sample is cut and weighed, immediately set up the analysis. Waiting to weigh out all sectors from roots collected from one field is what makes you need to leave sectors standing in the air. You may be tempted to do this when you want to incubate the samples at the same time but this can come at a cost of losing cyanide from the already prepared sectors. The time that samples are incubated can be maintained by taking note of samples prepared within 30 minute intervals. You will have to take note of the first and last sample prepared within each 30 minute period. All samples prepared in one 30 minutes interval should be removed from incubation at the same time. Doing this gives the same convenience with better results.

$4 \quad$ Add $0.5 \mathrm{ml}$ of clean water using the plastic pipette.

Notes:

- Use bottled water for this step if you have no access to distilled water. Tap water may be too variable in composition from one day to the next in some parts of the world. This variability 
would not be good if you are analysing cyanide in samples over several days.

5 Immediately add a yellow picrate paper attached to a plastic strip. The picrate paper must not touch the liquid in the bottle.

Notes:

- One side of the plastic strip on which the yellow picrate paper is attached is longer than the other. Make sure that this longer side is placed first into the bottle. This helps keeps the water in the bottle further away from the picrate paper. Remember that picrate papers must be always kept dry. Do not be afraid to place the picrate paper attached to the plastic strip into the bottle, it has been designed to perfectly fit into the bottle. However, be gentle as the water in the bottle may splash onto the picrate paper if you are not careful.

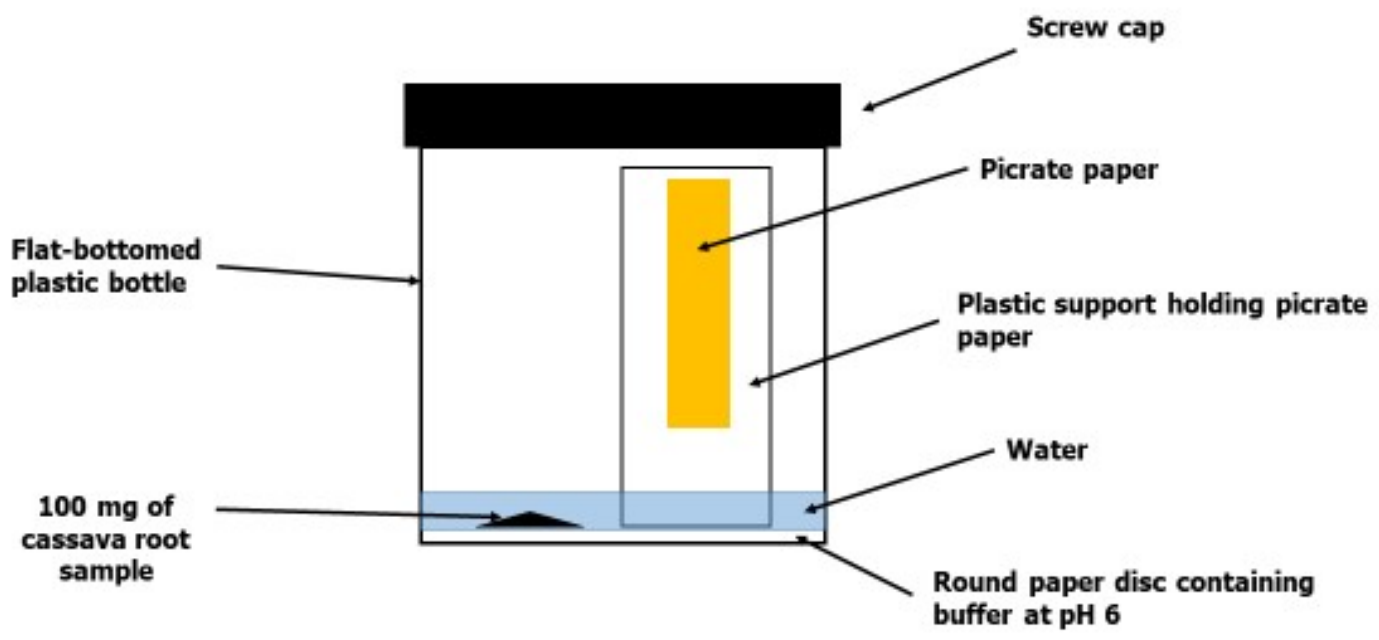

Figure 2. Set up for HCN capture from cassava roots suing the picrate paper method

6 IMMEDIATELY close the bottle with a screw capped lid.

7 As a blank, prepare another sample but with no cassava present. 
8 Every time you run a set of experiments you should check that the method works OK, using a standard pink linamarin paper from the kit.

9 Place a round paper disc with a black spot containing buffer and enzyme in the bottle. Add a pink standard paper and then $0.5 \mathrm{ml}$ water from a pipette and the picrate paper. IMMEDIATELY close the bottle with the screw capped lid.

10 Allow the bottles to stand for $16-24$ hour at room temperature.

Notes:

- This is the incubation step. This step should be carried out in the dark. You could use a cupboard for this. Just ensure that you arrange the picrate bottles in manner that will not block you from easily accessing any of them.

- You can take note of the hourly changes in temperatures. On cooler days you could decide to leave the picrate papers longer i.e. for the full 24 hours. 
Figure 3. Samples left to incubate in the dark

11 Open the bottles and match the colour of the picrate papers against the shades of colour of the colour chart supplied.

Notes:

- Once you open the bottles, carefully remove the picrate papers, still attached to the plastic strip, and place them on the plastic cap or on A4 sized paper (Figs. 4 and 5). This is done to allow the picrate papers to first dry-up. Picrate papers become a bit moist during incubation and must not be touched or made to brush against anything before they dry (and avoid directly touching them even when they are dry). Use the plastic strips to hold the picrate papers.

- The plastic caps are used for drying when you do not have to immediately reuse the plastic bottles. Using A4 papers for drying allows you to continue with other samples needing the bottles.

- To easily remember the identification of samples that have been left to dry on the A4 paper, place the 12 picrate papers belonging to the same field on the same A4 paper and arrange them in a manner that will help you to remember their order. You must place the field label on the A4 paper.

- Once dry you should label the picrate papers, by placing their ID mark on the plastic sheet (Fig. 
$5)$.

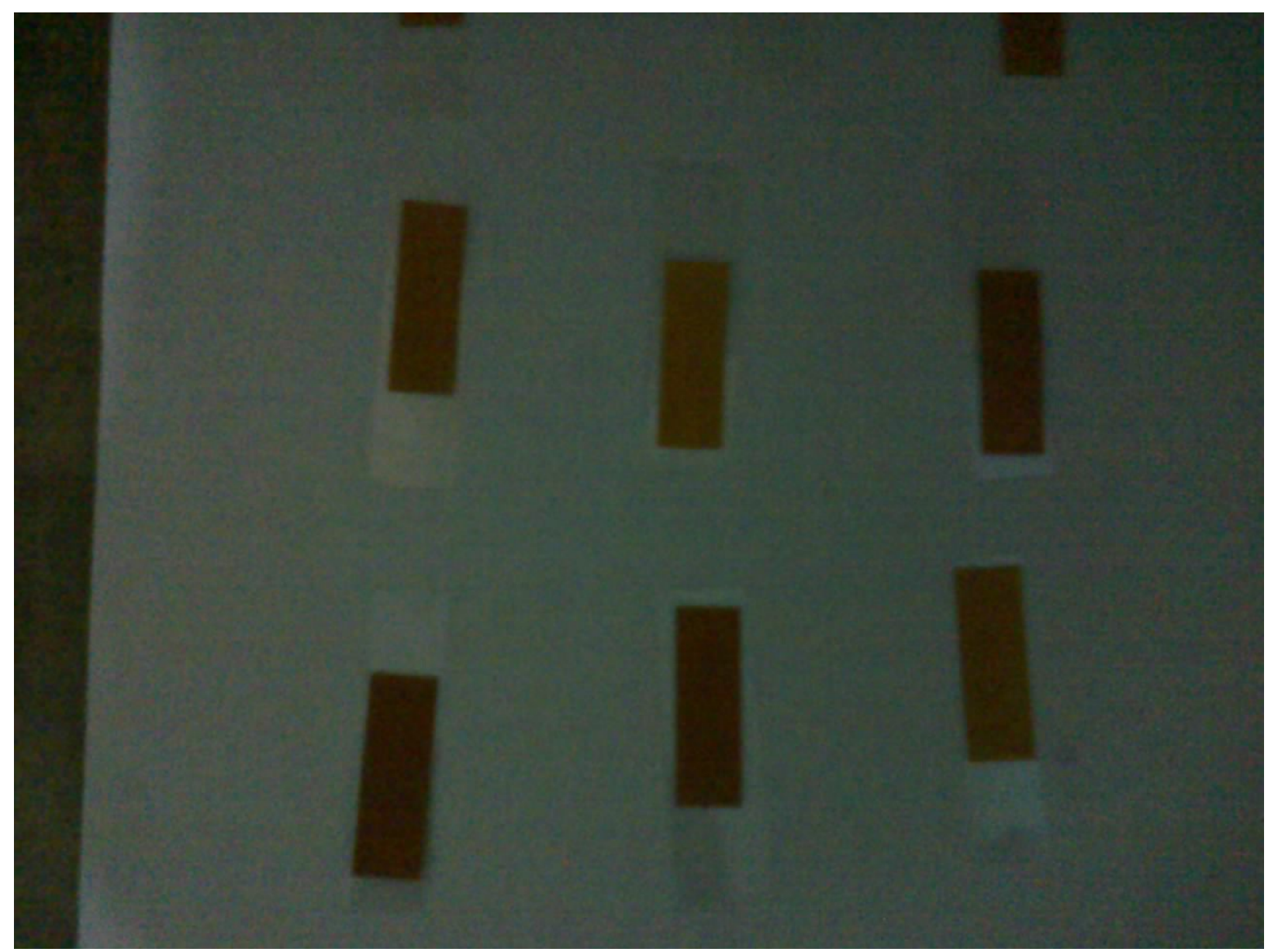

Figure 4. Picrate papers left to dry on A4 size paper and still attached on their plastic strip 


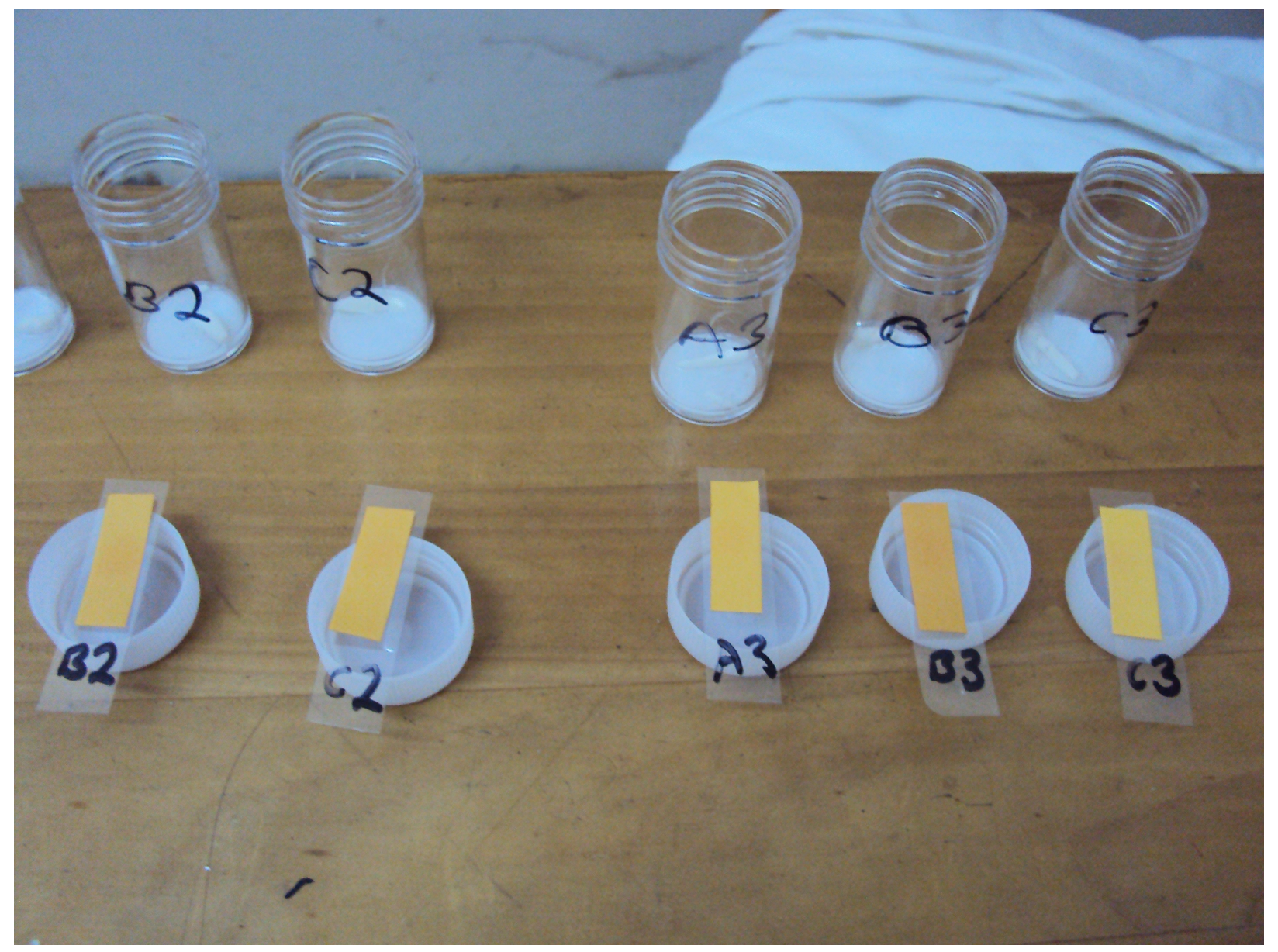

Figure 5: Labelled picrate papers that were left to dry on screw cap lids

12 Read off from the colour chart the total cyanide content in ppm in the cassava root. Also check out that the blank is zero and the standard gives a colour of about 50 ppm.

Notes:

- Colour chart readings are often the same as spectrophotometer readings and are usually just as reliable. If you are not sure of how to take the chart readings then consider using the spectrophotometer. It is ultimately better to get readings from a spectrophotometer.

- When taking colour chart readings, do not remove the picrate papers from the plastic strips if you plan to later get spectrophotometer readings from the same picrate papers. Do not however compare the reacted picrate papers by comparing the side covered by the plastic strip with the colour chart. It is the uncovered reacted picrate paper that must be used to get the colour reading. This is why it is advised to remove the plastic strip from the picrate paper when taking colour chart readings. You might however risk losing the label on the plastic strip if you remove the picrate paper from the plastic strip.

- If you have to travel to a research lab to use a spectrophotometer, then you will have to prepare the picrate papers for transportation. It is advisable to still take the colour reading in case something accidentally happens to the picrate papers while in transit. To better preserve 
them, picrate papers they will need to be individually placed in small plastic bags. They could also be individually wrapped in plastic. Gladwrap can be used for this purpose. Remember to group individually wrapped reacted picrate papers from the same field together. Remember to still keep the picrate papers away from light and do not also expose them to room temperatures for long. The firm opaque storage container and cool box will again be handy. Do the transportation as quickly as possible and immediately place the picrate papers still in the storage container in the deep freezer once you arrive at your destination. Although picrate papers can stay stable indefinitely in the deep freezer try to carry out the spectrophotometer reading within $1-2$ days. Storing reacted picrate papers in this manner is also necessary when you have to wait to take spectrophotometer readings, despite working from a lab.

\section{USING THE SPECTROPHOTOMETER TO DETERMINE ROOT HC.}

13 Carefully remove the plastic backing sheet (it may be washed and used again) from the picrate paper.

14 Place the picrate paper in a test tube and add $5.0 \mathrm{ml}$ of water measured accurately with a pipette.

\section{Notes:}

- 25 - $50 \mathrm{ml}$ beakers can also be used instead of test-tubes. Unlike test-tubes they are wider and picrate papers can be easily removed from them. Swirling is also easier in beakers. However, getting several beakers of this size may be difficult thus any lab ware that can be used as a beaker can be alternatively used.

- Be careful not to spill out any of the solution while swirling the contents.

15 Leave the test tube at room temperature for about $\mathbf{3 0}$ min with occasional gentle stirring.

\section{Notes:}

- After the 30 minutes have elapsed remove the picrate paper from the test-tube or beaker using a plastic disposable knife. The picrate paper easily attaches to the plastic knife. Do not use something metallic or something with a suction capability. Remember to rinse the knife in distilled water as you move from one beaker to the next.

16 Take the blank picrate paper (see 7 above), remove its plastic sheet and place the yellow picrate paper in $5.0 \mathrm{ml}$ of water for about $\mathbf{3 0} \mathrm{min}$ with occasional gentle stirring.

17 Measure the absorbance at $510 \mathrm{~nm}$ of the picrate solution from 15 against the blank from 16. 
18 The total cyanide content in ppm is calculated by the Equation 1

Total cyanide content $(\mathrm{ppm})$ fresh weight $=396 \times$ absorbance $\ldots .$. Equation 1

19 The cyanide content obtained for the same sample of root, from both measurements 12 and 18, should be about the same. Also check that the standard value agrees using both methods.

\section{BIBLIOGRAPHY}

20 1. Egan SV, Yeoh HH, Bradbury JH. Simple picrate paper kit for determination of the cyanogenic potential of cassava flour. J Sci Food Agric. 1998;76: 39-48.

2. Bradbury MG, Egan SV, Bradbury JH. Picrate paper kits for determination of total cyanogens in cassava roots and all forms of cyanogens in cassava products. J Sci Food Agric. 1999;79:

$593-601$. 